\title{
A shifted Jacobi-Gauss-Lobatto collocation method for solving nonlinear fractional Langevin equation involving two fractional orders in different intervals
}

\author{
Ali H Bhrawy ${ }^{1,2^{*}}$ and Mohammed A Alghamdi ${ }^{1}$
}

${ }^{*}$ Correspondence:

alibhrawy@yahoo.co.uk

'Department of Mathematics,

Faculty of Science, King Abdulaziz

University, Jeddah 21589, Saudi

Arabia

${ }^{2}$ Department of Mathematics,

Faculty of Science, Beni-Suef

University, Beni-Suef, Egypt

\begin{abstract}
In this paper, we develop a Jacobi-Gauss-Lobatto collocation method for solving the nonlinear fractional Langevin equation with three-point boundary conditions. The fractional derivative is described in the Caputo sense. The shifted Jacobi-Gauss-Lobatto points are used as collocation nodes. The main characteristic behind the Jacobi-Gauss-Lobatto collocation approach is that it reduces such a problem to those of solving a system of algebraic equations. This system is written in a compact matrix form. Through several numerical examples, we evaluate the accuracy and performance of the proposed method. The method is easy to implement and yields very accurate results.
\end{abstract}

Keywords: fractional Langevin equation; three-point boundary conditions; collocation method; Jacobi-Gauss-Lobatto quadrature; shifted Jacobi polynomials

\section{Introduction}

Many practical problems arising in science and engineering require solving initial and boundary value problems of fractional order differential equations (FDEs), see [1, 2] and references therein. Several methods have also been proposed in the literature to solve FDEs (see, for instance, [3-7]). Spectral methods are relatively new approaches to provide an accurate approximation to FDEs (see, for instance, [8-11]).

In this work, we propose the shifted Jacobi-Gauss-Lobatto collocation (SJ-GL-C) method to solve numerically the following nonlinear Langevin equation involving two fractional orders in different intervals:

$$
D^{v}\left(D^{\mu}+\lambda\right) u(x)=f(x, u(x)), \quad 0<\mu \leq 1,1<v \leq 2, x \in I=[0, L],
$$

subject to the three-point boundary conditions

$$
\left.u(0)=s_{0}, \quad u\left(x_{1}\right)=s_{1}, \quad u(L)=s_{2}, \quad x_{1} \in\right] 0, L[,
$$

where $D^{v} u(x) \equiv u^{(v)}(x)$ denotes the Caputo fractional derivative of order $v$ for $u(x), \lambda$ is a real number, $s_{0}, s_{1}, s_{2}$ are given constants and $f$ is a given nonlinear source function.

(c) 2012 Bhrawy and Alghamdi; licensee Springer. This is an Open Access article distributed under the terms of the Creative Commons Attribution License (http://creativecommons.org/licenses/by/2.0), which permits unrestricted use, distribution, and reproduction in any medium, provided the original work is properly cited. 
The existence and uniqueness of solution of Langevin equation involving two fractional orders in different intervals $(0<\mu \leq 1,1<v \leq 2)$ have been studied in [12], and for other choices of $v$ and $\mu$, see $[13,14]$.

Fractional Langevin equation is one of the basic equations in the theory of the evolution of physical phenomena in fluctuating environments and provides a more flexible model for fractal processes as compared with the usual ordinary Langevin equation. Moreover, fractional generalized Langevin equation with external force is used to model single-file diffusion. This equation has been the focus of many studies, see, for instance, [15-18].

Due to high order accuracy, spectral methods have gained increasing popularity for several decades, especially in the field of computational fluid dynamics (see, e.g., [19] and the references therein). Collocation methods have become increasingly popular for solving differential equations; also, they are very useful in providing highly accurate solutions to nonlinear differential equations [20-22]. Bhrawy and Alofi [20] proposed the spectral shifted Jacobi-Gauss collocation method to find the solution of the Lane-Emden type equation. Moreover, Doha et al. [23] developed the shifted Jacobi-Gauss collocation method for solving nonlinear high-order multi-point boundary value problems. To the best of our knowledge, there are no results on Jacobi-Gauss-Lobatto collocation method for three-point nonlinear Langevin equation arising in mathematical physics. This partially motivated our interest in such a method.

The advantage of using Jacobi polynomials for solving differential equations is obtaining the solution in terms of the Jacobi parameters $\alpha$ and $\beta$ (see [24-27]). Some special cases of Jacobi parameters $\alpha$ and $\beta$ are used for numerically solving various types of differential equations (see [28-31]).

The main concern of this paper is to extend the application of collocation method to solve the three-point nonlinear Langevin equation involving two fractional orders in different intervals. It would be very useful to carry out a systematic study on Jacobi-GaussLobatto collocation method with general indexes $(\alpha, \beta>-1)$. The fractional Langevin equation is collocated only at $(N-2)$ points; for suitable collocation points, we use the $(N-2)$ nodes of the shifted Jacobi-Gauss-Lobatto interpolation $(\alpha, \beta>-1)$. These equations together with the three-point boundary conditions generate $(N+1)$ nonlinear algebraic equations which can be solved using Newton's iterative method. Finally, the accuracy of the proposed method is demonstrated by test problems.

The remainder of the paper is organized as follows. In the next section, we introduce some notations and summarize a few mathematical facts used in the remainder of the paper. In Section 3, the way of constructing the Gauss-Lobatto collocation technique for fractional Langevin equation is described using the shifted Jacobi polynomials; and in Section 4 the proposed method is applied to some types of Langevin equations. Finally, some concluding remarks are given in Section 5.

\section{Preliminaries}

In this section, we give some definitions and properties of the fractional calculus (see, e.g., $[1,2,32])$ and Jacobi polynomials (see, e.g., [33-35]). 
Definition 2.1 The Riemann-Liouville fractional integral operator of order $\mu(\mu \geq 0)$ is defined as

$$
\begin{aligned}
& J^{\mu} f(x)=\frac{1}{\Gamma(\mu)} \int_{0}^{x}(x-t)^{\mu-1} f(t) d t, \quad \mu>0, x>0, \\
& J^{0} f(x)=f(x) .
\end{aligned}
$$

Definition 2.2 The Caputo fractional derivative of order $\mu$ is defined as

$$
\begin{aligned}
D^{\mu} f(x) & =J^{m-\mu} D^{m} f(x)=\frac{1}{\Gamma(m-\mu)} \int_{0}^{x}(x-t)^{m-\mu-1} \frac{d^{m}}{d t^{m}} f(t) d t, \\
m-1 & <\mu \leq m, x>0,
\end{aligned}
$$

where $m$ is an integer number and $D^{m}$ is the classical differential operator of order $m$.

For the Caputo derivative, we have

$$
D^{\mu} x^{\beta}= \begin{cases}0, & \text { for } \beta \in N_{0} \text { and } \beta<\lceil\mu\rceil, \\ \frac{\Gamma(\beta+1)}{\Gamma(\beta+1-\mu)} x^{\beta-\mu}, & \text { for } \beta \in N_{0} \text { and } \beta \geq\lceil\mu\rceil \text { or } \beta \notin N \text { and } \beta>\lfloor\mu\rfloor .\end{cases}
$$

We use the ceiling function $\lceil\mu\rceil$ to denote the smallest integer greater than or equal to $\mu$ and the floor function $\lfloor\mu\rfloor$ to denote the largest integer less than or equal to $\mu$. Also $N=\{1,2, \ldots\}$ and $N_{0}=\{0,1,2, \ldots\}$. Recall that for $\mu \in N$, the Caputo differential operator coincides with the usual differential operator of an integer order.

Let $\alpha>-1, \beta>-1$ and $P_{k}^{(\alpha, \beta)}(x)$ be the standard Jacobi polynomial of degree $k$. We have that

$$
\begin{aligned}
& P_{k}^{(\alpha, \beta)}(-x)=(-1)^{k} P_{k}^{(\alpha, \beta)}(x), \quad P_{k}^{(\alpha, \beta)}(-1)=\frac{(-1)^{k} \Gamma(k+\beta+1)}{k ! \Gamma(\beta+1)}, \\
& P_{k}^{(\alpha, \beta)}(1)=\frac{\Gamma(k+\alpha+1)}{k ! \Gamma(\alpha+1)} .
\end{aligned}
$$

Besides,

$$
D^{m} P_{k}^{(\alpha, \beta)}(x)=2^{-m} \frac{\Gamma(m+k+\alpha+\beta+1)}{\Gamma(k+\alpha+\beta+1)} P_{k-m}^{(\alpha+m, \beta+m)}(x) .
$$

Let $w^{(\alpha, \beta)}(x)=(1-x)^{\alpha}(1+x)^{\beta}$, then we define the weighted space $L_{w^{(\alpha, \beta)}}^{2}(-1,1)$ as usual, equipped with the following inner product and norm:

$$
(u, v)_{w^{(\alpha, \beta)}}=\int_{-1}^{1} u(x) v(x) w^{(\alpha, \beta)}(x) d x, \quad\|v\|_{w^{(\alpha, \beta)}}=(v, v)_{w^{(\alpha, \beta)}}^{\frac{1}{2}} .
$$

The set of Jacobi polynomials forms a complete $L_{w^{\alpha, \beta}}^{2}(-1,1)$-orthogonal system, and

$$
\left\|P_{k}^{(\alpha, \beta)}\right\|_{w^{(\alpha, \beta)}}^{2}=h_{k}^{(\alpha, \beta)}=\frac{2^{\alpha+\beta+1} \Gamma(k+\alpha+1) \Gamma(k+\beta+1)}{(2 k+\alpha+\beta+1) \Gamma(k+1) \Gamma(k+\alpha+\beta+1)} .
$$


Let $L>0$, then the shifted Jacobi polynomial of degree $k$ on the interval $(0, L)$ is defined by $P_{L, k}^{(\alpha, \beta)}(x)=P_{k}^{(\alpha, \beta)}\left(\frac{2 x}{L}-1\right)$.

By virtue of (6), we have that

$$
P_{L, j}^{(\alpha, \beta)}(0)=(-1)^{j} \frac{\Gamma(j+\beta+1)}{\Gamma(\beta+1) j !} .
$$

Next, let $w_{L}^{(\alpha, \beta)}(x)=(L-x)^{\alpha} x^{\beta}$, then we define the weighted space $L_{w_{L}^{(\alpha, \beta)}}^{2}(0, L)$ in the usual way, with the following inner product and norm:

$$
(u, v)_{w_{L}^{(\alpha, \beta)}}=\int_{0}^{L} u(x) v(x) w_{L}^{(\alpha, \beta)}(x) d x, \quad\|v\|_{w_{L}^{(\alpha, \beta)}}=(v, v)_{w_{L}^{(\alpha, \beta)}}^{\frac{1}{2}} .
$$

The set of shifted Jacobi polynomials is a complete $L_{w_{L}^{(\alpha, \beta)}}^{2}(0, L)$-orthogonal system. Moreover, due to (8), we have

$$
\left\|P_{L, k}^{(\alpha, \beta)}\right\|_{w_{L}^{(\alpha, \beta)}}^{2}=\left(\frac{L}{2}\right)^{\alpha+\beta+1} h_{k}^{(\alpha, \beta)}=h_{L, k}^{(\alpha, \beta)}
$$

For $\alpha=\beta$ one recovers the shifted ultraspherical polynomials (symmetric shifted Jacobi polynomials) and for $\alpha=\beta=\mp \frac{1}{2}, \alpha=\beta=0$, the shifted Chebyshev of the first and second kinds and shifted Legendre polynomials respectively; and for the nonsymmetric shifted Jacobi polynomials, the two important special cases $\alpha=-\beta= \pm \frac{1}{2}$ (shifted Chebyshev polynomials of the third and fourth kinds) are also recovered.

\section{Shifted Jacobi-Gauss-Lobatto collocation method}

In this section, we derive the SJ-GL-C method to solve numerically the following model problem:

$$
D^{v}\left(D^{\mu}+\lambda\right) u(x)=f(x, u), \quad 0<\mu \leq 1,1<v \leq 2, x \in I=(0, L),
$$

subject to the three-point boundary conditions

$$
\left.u(0)=s_{0}, \quad u\left(x_{1}\right)=s_{1}, \quad u(L)=s_{2}, \quad x_{1} \in\right] 0, L[,
$$

where $D^{v} u(x) \equiv u^{(v)}(x)$ denotes the Caputo fractional derivative of order $v$ for $u(x), \lambda$ is a real number, $s_{0}, s_{1}, s_{2}$ are given constants and $f(x, u)$ is a given nonlinear source function. For the existence and uniqueness of solution of (11)-(12), see [12].

The choice of collocation points is important for the convergence and efficiency of the collocation method. For boundary value problems, the Gauss-Lobatto points are commonly used. It should be noted that for a differential equation with the singularity at $x=0$ in the interval $[0, L]$ one is unable to apply the collocation method with Jacobi-GaussLobatto points because the two assigned abscissas 0 and $L$ are necessary to use as a two points from the collocation nodes. Also, a Jacobi-Gauss-Radau nodes with the fixed node $x=0$ cannot be used in this case. In fact, we use the collocation method with JacobiGauss-Lobatto nodes to treat the nonlinear Langevin differential equation; i.e., we collocate this equation only at the $(N-2)$ Jacobi-Gauss-Lobatto points $(0, L)$. These equations 
together with three-point boundary conditions generate $(N+1)$ nonlinear algebraic equations which can be solved.

Let us first introduce some basic notation that will be used in the sequel. We set

$$
S_{N}(0, L)=\operatorname{span}\left\{P_{L, 0}^{(\alpha, \beta)}(x), P_{L, 1}^{(\alpha, \beta)}(x), \ldots, P_{L, N}^{(\alpha, \beta)}(x)\right\}
$$

We next recall the Jacobi-Gauss-Lobatto interpolation. For any positive integer $N, S_{N}(0, L)$ stands for the set of all algebraic polynomials of degree at most $N$. If we denote by $x_{N, j}^{(\alpha, \beta)}\left(x_{L, N, j}^{(\alpha, \beta)}\right), 0 \leq j \leq N$, and $\varpi_{N, j}^{(\alpha, \beta)}\left(\varpi_{L, N, j}^{(\alpha, \beta)}\right),(0 \leq i \leq N)$, to the nodes and Christoffel numbers of the standard (shifted) Jacobi-Gauss-Lobatto quadratures on the intervals $(-1,1)$, $(0, L)$ respectively. Then one can easily show that

$$
\begin{aligned}
& x_{L, N, j}^{(\alpha, \beta)}=\frac{L}{2}\left(x_{N, j}^{(\alpha, \beta)}+1\right), \quad 0 \leq j \leq N, \\
& \varpi_{L, N, j}^{(\alpha, \beta)}=\left(\frac{L}{2}\right)^{\alpha+\beta+1} \varpi_{N, j}^{(\alpha, \beta)}, \quad 0 \leq j \leq N .
\end{aligned}
$$

For any $\phi \in S_{2 N+1}(0, L)$,

$$
\begin{aligned}
\int_{0}^{L} w_{L}^{(\alpha, \beta)}(x) \phi(x) d x & =\left(\frac{L}{2}\right)^{\alpha+\beta+1} \int_{-1}^{1}(1-x)^{\alpha}(1+x)^{\beta} \phi\left(\frac{L}{2}(x+1)\right) d x \\
& =\left(\frac{L}{2}\right)^{\alpha+\beta+1} \sum_{j=0}^{N} \varpi_{N, j}^{(\alpha, \beta)} \phi\left(\frac{L}{2}\left(x_{N, j}^{(\alpha, \beta)}+1\right)\right) \\
& =\sum_{j=0}^{N} \varpi_{L, N, j}^{(\alpha, \beta)} \phi\left(x_{L, N, j}^{(\alpha, \beta)}\right) .
\end{aligned}
$$

We introduce the following discrete inner product and norm:

$$
(u, v)_{w_{L}^{(\alpha, \beta)}, N}=\sum_{j=0}^{N} u\left(x_{L, N, j}^{(\alpha, \beta)}\right) v\left(x_{L, N, j}^{(\alpha, \beta)}\right) \varpi_{L, N, j}^{(\alpha, \beta)}, \quad\|u\|_{w_{L}^{(\alpha, \beta)}, N}=\sqrt{(u, u)_{w_{L}^{(\alpha, \beta)}, N}},
$$

where $x_{L, N, j}^{(\alpha, \beta)}$ and $\varpi_{L, N, j}^{(\alpha, \beta)}$ are the nodes and the corresponding weights of the shifted JacobiGauss-quadrature formula on the interval $(0, L)$ respectively.

Due to (14), we have

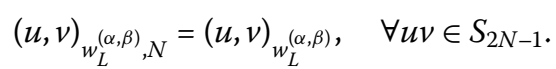

Thus, for any $u \in S_{N}(0, L)$, the norms $\|u\|_{w_{L}^{(\alpha, \beta)}, N}$ and $\|u\|_{w_{L}^{(\alpha, \beta)}}$ coincide.

Associating with this quadrature rule, we denote by $I_{N}^{P_{L}^{(\alpha, \beta)}}$ the shifted Jacobi-Gauss interpolation,

$$
I_{N}^{P_{L}^{(\alpha, \beta)}} u\left(x_{L, N, j}^{(\alpha, \beta)}\right)=u\left(x_{L, N, j}^{(\alpha, \beta)}\right), \quad 0 \leq k \leq N
$$


The shifted Jacobi-Gauss collocation method for solving (11)-(12) is to seek $u_{N}(x) \in$ $S_{N}(0, T)$, such that

$$
\begin{aligned}
& D^{\mu+v} u_{N}\left(x_{L, N-3, k}^{(\alpha, \beta)}\right)+\lambda D^{v} u_{N}\left(x_{L, N-3, k}^{(\alpha, \beta)}\right) \\
& \quad=f\left(x_{L, N-3, k}^{(\alpha, \beta)}, u_{N}\left(x_{L, N-3, k}^{(\alpha, \beta)}\right)\right), \quad k=0,1, \ldots, N-3 . \\
& \left.u_{N}(0)=s_{0}, \quad u_{N}\left(x_{1}\right)=s_{1}, \quad u_{N}(L)=s_{2}, \quad x_{1} \in\right] 0, L[.
\end{aligned}
$$

We now derive an efficient algorithm for solving (17)-(18). Let

$$
u_{N}(x)=\sum_{j=0}^{N} a_{j} P_{L, j}^{(\alpha, \beta)}(x), \quad \mathbf{a}=\left(a_{0}, a_{1}, \ldots, a_{N}\right)^{T}
$$

We first approximate $u(x), D^{\mu+v} u(x)$ and $D^{\mu} u(x)$, as Eq. (19). By substituting these approximations in Eq. (11), we get

$$
\sum_{j=0}^{N} a_{j}\left(D^{\mu+v} P_{L, j}^{(\alpha, \beta)}(x)+\lambda D^{\mu} P_{L, j}^{(\alpha, \beta)}(x)\right)=f\left(x, \sum_{j=0}^{N} a_{j} P_{L, j}^{(\alpha, \beta)}(x)\right)
$$

Here, the fractional derivative of order $\mu$ in the Caputo sense for the shifted Jacobi polynomials expanded in terms of shifted Jacobi polynomials themselves can be represented formally in the following theorem.

Theorem 3.1 Let $P_{L, j}^{(\alpha, \beta)}(x)$ be a shifted Jacobi polynomial of degree $j$, then the fractional derivative of order $v$ in the Caputo sense for $P_{L, j}^{(\alpha, \beta)}(x)$ is given by

$$
D^{\nu} P_{L, j}^{(\alpha, \beta)}(x)=\sum_{i=0}^{\infty} Q_{\nu}(j, i, \alpha, \beta) P_{L, i}^{(\alpha, \beta)}(x), \quad j=\lceil\nu\rceil,\lceil\nu\rceil+1, \ldots,
$$

where

$$
\begin{aligned}
Q_{v}(j, i, \alpha, \beta)= & \sum_{k=\lceil v\rceil}^{j} \frac{(-1)^{j-k} L^{\alpha+\beta-v+1} \Gamma(i+\beta+1) \Gamma(j+\beta+1) \Gamma(j+k+\alpha+\beta+1)}{h_{i} \Gamma(i+\alpha+\beta+1) \Gamma(k+\beta+1) \Gamma(j+\alpha+\beta+1) \Gamma(k-v+1)(j-k) !} \\
& \times \sum_{l=0}^{i} \frac{(-1)^{i-l} \Gamma(i+l+\alpha+\beta+1) \Gamma(\alpha+1) \Gamma(l+k+\beta-v+1)}{\Gamma(l+\beta+1) \Gamma(l+k+\alpha+\beta-v+2)(i-l) ! l !} .
\end{aligned}
$$

Proof This theorem can be easily proved (see Doha et al. [36]).

In practice, only the first $(N+1)$-terms shifted Jacobi polynomials are considered, with the aid of Theorem 3.1 (Eq. (21)), we obtain from (20) that

$$
\begin{aligned}
& \sum_{j=0}^{N} a_{j}\left(\sum_{i=0}^{N} Q_{\mu+\nu}(j, i, \alpha, \beta) P_{L, i}^{(\alpha, \beta)}(x)+\lambda \sum_{i=0}^{N} Q_{\mu}(j, i, \alpha, \beta) P_{L, i}^{(\alpha, \beta)}(x)\right) \\
& \quad=f\left(x, \sum_{j=0}^{N} a_{j} P_{L, j}^{(\alpha, \beta)}(x)\right) .
\end{aligned}
$$


Also, by substituting Eq. (19) in Eq. (12) we obtain

$$
\left.\begin{array}{l}
\sum_{j=0}^{N} a_{j} P_{L, j}^{(\alpha, \beta)}(0)=s_{0}, \\
\sum_{j=0}^{N} a_{j} P_{L, j}^{(\alpha, \beta)}\left(x_{1}\right)=s_{1}, \\
\sum_{j=0}^{N} a_{j} P_{L, j}^{(\alpha, \beta)}(L)=s_{2} .
\end{array}\right\}
$$

To find the solution $u_{N}(x)$, we first collocate Eq. (22) at the $(N-2)$ shifted Jacobi-GaussLobatto notes, yields

$$
\begin{aligned}
& \sum_{j=0}^{N} a_{j}\left(\sum_{i=0}^{N} Q_{\mu+v}(j, i, \alpha, \beta) P_{L, i}^{(\alpha, \beta)}\left(x_{L, N-3, k}^{(\alpha, \beta)}\right)+\lambda \sum_{i=0}^{N} Q_{\mu}(j, i, \alpha, \beta) P_{L, i}^{(\alpha, \beta)}\left(x_{L, N-3, k}^{(\alpha, \beta)}\right)\right) \\
& =f\left(\left(x_{L, N-3, k}^{(\alpha, \beta)}\right), \sum_{j=0}^{N} a_{j} P_{L, j}^{(\alpha, \beta)}\left(x_{L, N-3, k}^{(\alpha, \beta)}\right), \quad 0 \leq k \leq N-3 .\right.
\end{aligned}
$$

Next, Eq. (23), after using (9) and (6), can be written as

$$
\left.\begin{array}{r}
\sum_{j=0}^{N}(-1)^{j} \frac{\Gamma(j+\beta+1)}{\Gamma(\beta+1) j !} a_{j}=s_{0}, \\
\sum_{j=0}^{N}\left(\sum_{i=0}^{j}(-1)^{j-i} \frac{\Gamma(j+\beta+1) \Gamma(j+i+\alpha+\beta+1)}{\Gamma(i+\beta+1) \Gamma(j+\alpha+\beta+1)(j-i) ! i ! L^{i}} x_{1}^{i}\right) a_{j}=s_{1}, \\
\sum_{j=0}^{N}\left(\sum_{i=0}^{j}(-1)^{j-i} \frac{\Gamma(j+\beta+1) \Gamma(j+i+\alpha+\beta+1)}{\Gamma(i+\beta+1) \Gamma(j+\alpha+\beta+1)(j-i) ! i !}\right) a_{j}=s_{2} .
\end{array}\right\}
$$

The scheme (24)-(25) can be rewritten as a compact matrix form. To do this, we introduce the $(N+1) \times(N+1)$ matrix $A$ with the entries $a_{k j}$ as follows:

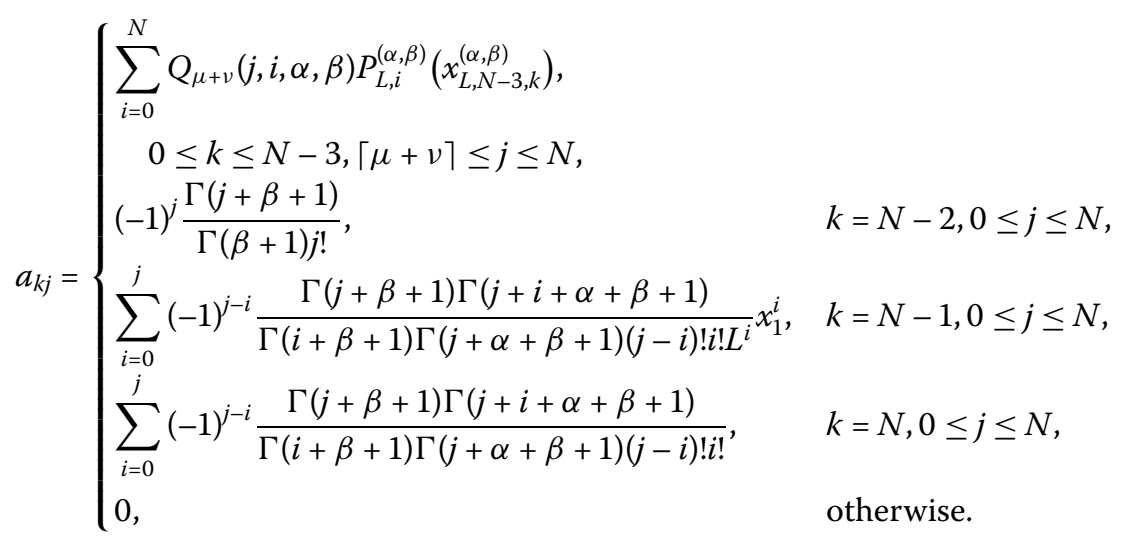


Also, we define the $(N+1) \times(N+1)$ matrix $B$ with the entries:

$$
b_{k j}= \begin{cases}\sum_{i=0}^{N} Q_{\mu}(j, i, \alpha, \beta) P_{L, i}^{(\alpha, \beta)}\left(x_{L, N-3, k}^{(\alpha, \beta)}\right), & 0 \leq k \leq N-3,\lceil\mu\rceil \leq j \leq N, \\ 0, & \text { otherwise, }\end{cases}
$$

and the $(N-2) \times(N+1)$ matrix $C$ with the entries:

$$
c_{k j}=P_{T, j}^{(\alpha, \beta)}\left(x_{T, N-3, k}^{(\alpha, \beta)}\right), \quad 0 \leq k \leq N-3,0 \leq j \leq N .
$$

Further, let $\mathbf{a}=\left(a_{0}, a_{1}, \ldots, a_{N}\right)^{T}$, and

$$
\mathbf{F}(\mathbf{a})=\left(f\left(x_{T, N-3,0}^{(\alpha, \beta)}, u_{N}\left(x_{T, N-3,0}^{(\alpha, \beta)}\right)\right), \ldots, f\left(x_{T, N-3, N-3}^{(\alpha, \beta)}, u_{N}\left(x_{T, N-3, N-3}^{(\alpha, \beta)}\right)\right), s_{0}, s_{1}, s_{2}\right)^{T},
$$

where $u_{N}\left(x_{T, N-3, k}^{(\alpha, \beta)}\right)$ is the $k$ th component of $C \mathbf{a}$. Then we obtain from (24)-(25) that

$$
(A+\lambda B) \mathbf{a}=\mathbf{F}(\mathbf{a}),
$$

or equivalently

$$
\mathbf{a}=(A+\lambda B)^{-1} \mathbf{F}(\mathbf{a}) .
$$

Finally, from (26), we obtain $(N+1)$ nonlinear algebraic equations which can be solved for the unknown coefficients $a_{j}$ by using any standard iteration technique, like Newton's iteration method. Consequently, $u_{N}(x)$ given in Eq. (19) can be evaluated.

Remark 3.2 In actual computation for fixed $\mu, v$ and $\lambda$, it is required to compute $(A+$ $\lambda B)^{-1}$ only once. This allows us to save a significant amount of computational time.

\section{Numerical results}

To illustrate the effectiveness of the proposed method in the present paper, two test examples are carried out in this section. Comparison of the results obtained by various choices of Jacobi parameters $\alpha$ and $\beta$ reveal that the present method is very effective and convenient for all choices of $\alpha$ and $\beta$.

We consider the following two examples.

Example 1 Consider the nonlinear fractional Langevin equation

$$
D^{\frac{7}{4}}\left(D^{\frac{3}{4}}+\frac{1}{8}\right) u(x)=\frac{18}{10}\left(\tan ^{-1} u(x)+\cos x\right), \quad \text { in } I=(0,1),
$$

subject to three-point boundary conditions:

$$
u(0)=0, \quad u(0.5)=0, \quad u(1)=0 .
$$

The analytic solution for this problem is not known. In Table 1 we introduce the approximate solution for (27)-(28) using SJ-GL-C method at $\alpha=\beta=0$ and $N=12$. The 
Table 1 Approximate solution of (27)-(28) using SJ-GL-C method for $N=12$

\begin{tabular}{lc}
\hline $\boldsymbol{x}$ & Approximate solution \\
\hline 0.1 & 0.00837437 \\
0.2 & 0.0101356 \\
0.3 & 0.00811427 \\
0.4 & 0.00430877 \\
0.5 & $-9.994 \times 10^{-20}$ \\
\hline
\end{tabular}

\begin{tabular}{ll}
\hline $\boldsymbol{x}$ & Approximate solution \\
\hline 0.6 & -0.00364602 \\
0.7 & -0.00585357 \\
0.8 & -0.00615727 \\
0.9 & -0.00421287 \\
1.0 & $6.098 \times 10^{-19}$ \\
\hline
\end{tabular}

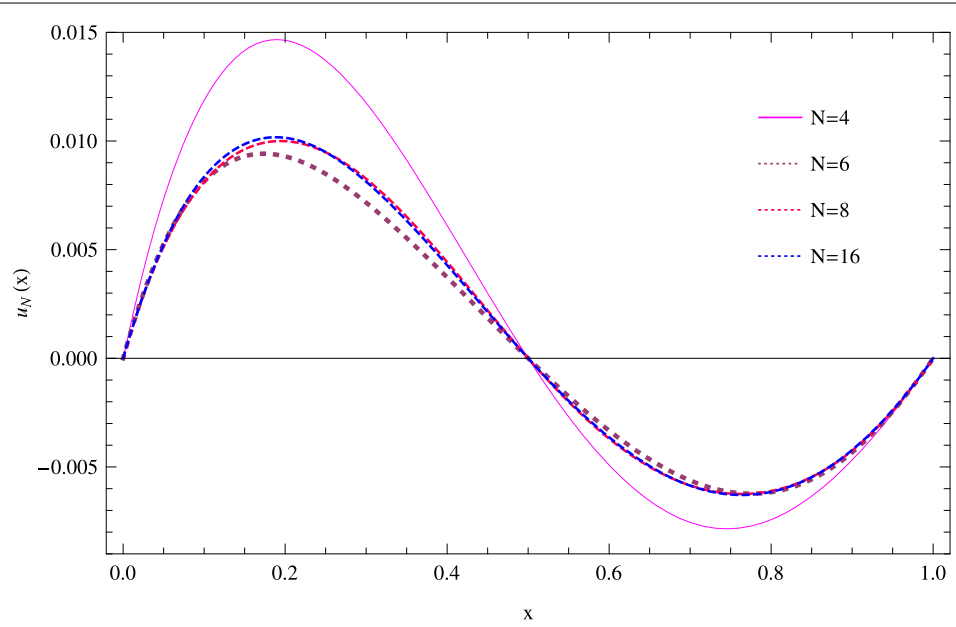

Figure 1 Comparing the approximate solutions at $N=4,6,8,16$, for Example 1 .

approximate solutions at $\alpha=\beta=-\frac{1}{2}$ and a few collocation points $(N=4,6,8,16)$ of this problem are depicted in Figure 1. The approximate solution at $N=8$ agrees very well with the approximate solution at $N=16$; this means the numerical solution converges fast as $N$ increases.

Example 2 In this example we consider the following nonlinear fractional Langevin differential equation

$$
D^{v}\left(D^{\mu}+3\right) u(x)=u^{3}(x)+e^{u(x)}+g(x), \quad v \in(1,2), \mu \in(0,1),
$$

subject to the following three-point boundary conditions:

$$
u(0)=0, \quad u\left(\frac{1}{3}\right)=\frac{729}{125,000}-\left(\frac{10}{3}\right)^{-2 \mu-\nu}, \quad u(1)=0
$$

where

$$
\begin{aligned}
g(x)= & -e^{3 x^{5}-2 x^{6}-x^{2 \mu+\nu}}-\left(3 x^{5}-2 x^{6}-x^{2 \mu+\nu}\right)^{3}+\frac{360 x^{5-\mu-\nu}}{\Gamma(6-\mu-\nu)}-\frac{1,440 x^{6-\mu-\nu}}{\Gamma(7-\mu-v)} \\
& -\frac{x^{\mu} \Gamma(1+2 \mu+\nu)}{\Gamma(1+\mu)}+3\left(\frac{360 x^{5-\nu}}{\Gamma(6-v)}-\frac{1,440 x^{6-\nu}}{\Gamma(7-v)}-\frac{x^{2 \mu} \Gamma(1+2 \mu+v)}{\Gamma(1+2 \mu)}\right) .
\end{aligned}
$$

The exact solution of this problem is $u(x)=-x^{\nu+2 \mu}+3 x^{5}-2 x^{6}$. 
Table 2 Maximum absolute error of $u-u_{N}$ using SJ-GL-C method for $\alpha=\beta=0$

\begin{tabular}{cccccc}
\hline $\boldsymbol{N}$ & $\boldsymbol{\alpha}$ & $\boldsymbol{\beta}$ & $\boldsymbol{v}=\mathbf{1 . 5}, \boldsymbol{\mu}=\mathbf{0 . 5}$ & $\boldsymbol{\nu}=\mathbf{1 . 8}, \boldsymbol{\mu}=\mathbf{0 . 8}$ & $\boldsymbol{\nu}=\mathbf{1 . 9 9 9}, \boldsymbol{\mu}=\mathbf{0 . 9 9 9}$ \\
\hline 8 & 0 & 0 & $2.09 \times 10^{-4}$ & $4.91 \times 10^{-5}$ & $1.07 \times 10^{-7}$ \\
16 & & & $1.39 \times 10^{-5}$ & $4.02 \times 10^{-7}$ & $3.99 \times 10^{-10}$ \\
24 & & & $3.25 \times 10^{-6}$ & $5.87 \times 10^{-8}$ & $2.33 \times 10^{-11}$ \\
\hline
\end{tabular}

Table 3 Maximum absolute error of $u-u_{N}$ using SJ-GL-C method for $\alpha=\beta=-1 / 2$

\begin{tabular}{rccccc}
\hline $\boldsymbol{N}$ & $\boldsymbol{\alpha}$ & $\boldsymbol{\beta}$ & $\boldsymbol{\nu}=\mathbf{1 . 5}, \boldsymbol{\mu = 0 . 5}$ & $\boldsymbol{\nu}=\mathbf{1 . 8}, \boldsymbol{\mu = 0 . 8}$ & $\boldsymbol{\nu}=\mathbf{1 . 9 9 9}, \boldsymbol{\mu}=\mathbf{0 . 9 9 9}$ \\
\hline 8 & $\frac{-1}{2}$ & $\frac{-1}{2}$ & $3.64 \times 10^{-4}$ & $1.15 \times 10^{-4}$ & $2.83 \times 10^{-7}$ \\
16 & & & $9.66 \times 10^{-6}$ & $1.16 \times 10^{-6}$ & $1.01 \times 10^{-9}$ \\
24 & & & $1.99 \times 10^{-6}$ & $8.35 \times 10^{-8}$ & $7.15 \times 10^{-11}$ \\
\hline
\end{tabular}

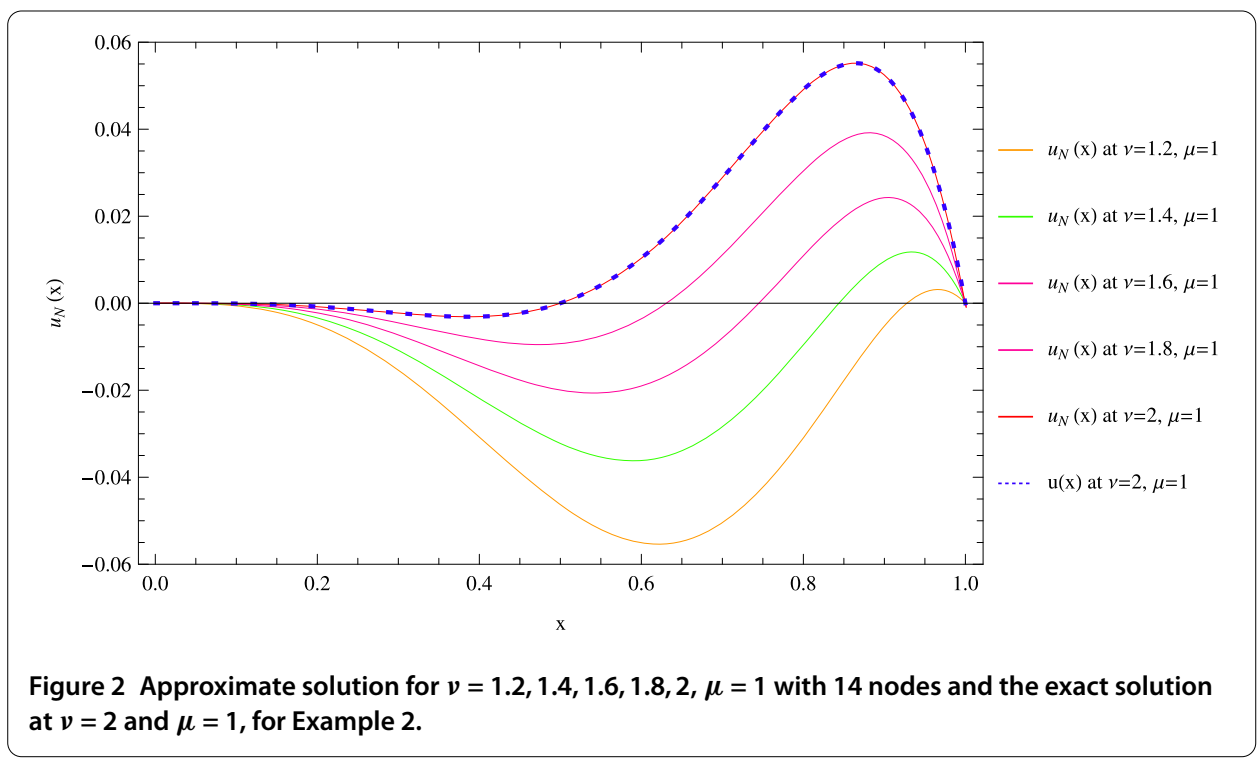

Numerical results are obtained for different choices of $\nu, \mu, \alpha, \beta$, and $N$. In Tables 2 and 3 we introduce the maximum absolute error, using the shifted Jacobi collocation method based on Gauss-Lobatto points, with two choices of $\alpha, \beta$, and various choices of $\nu, \mu$, and $N$.

The approximate solutions are evaluated for $\nu=1.2,1.4,1.6,1.8,2, \mu=1, \alpha=\beta=1$ and $N=14$. The results of the numerical simulations are plotted in Figure 2 . In Figure 3, we plotted the approximate solutions at fixed $\nu=2$, and various choices of $\mu=$ $0.2,0.4,0.6,0.8,1$ with $\alpha=\beta=1$ and $N=14$. It is evident from Figure 2 and Figure 3 that, as $\nu$ and $\mu$ approach close to 2 and 1 , the numerical solution by shifted Jacobi-Gauss-Lobatto collocation method with $\alpha=\beta=1$ for fractional order differential equation approaches to the solution of integer order differential equation.

In the case of $1<\nu \leq 2, \mu=1$ with $\alpha=\beta=\frac{1}{2}$, and $N=14$, the results of the numerical simulations are shown in Figure 4. In Figure 5, we plotted the approximate solutions for $v=2,0<\mu \leq 1$ with $\alpha=\beta=\frac{1}{2}$, and $N=14$. In fact, the approximate solutions obtained by the present method at $1<v \leq 2,0<\mu \leq 1$ with $N=14$ are shown in Figure 4 and Figure 5 to make it easier to show that; as $v$ and $\mu$ approach to their integer values, the solution of fractional order Langevin equation approaches to the solution of integer order Langevin differential equation. 

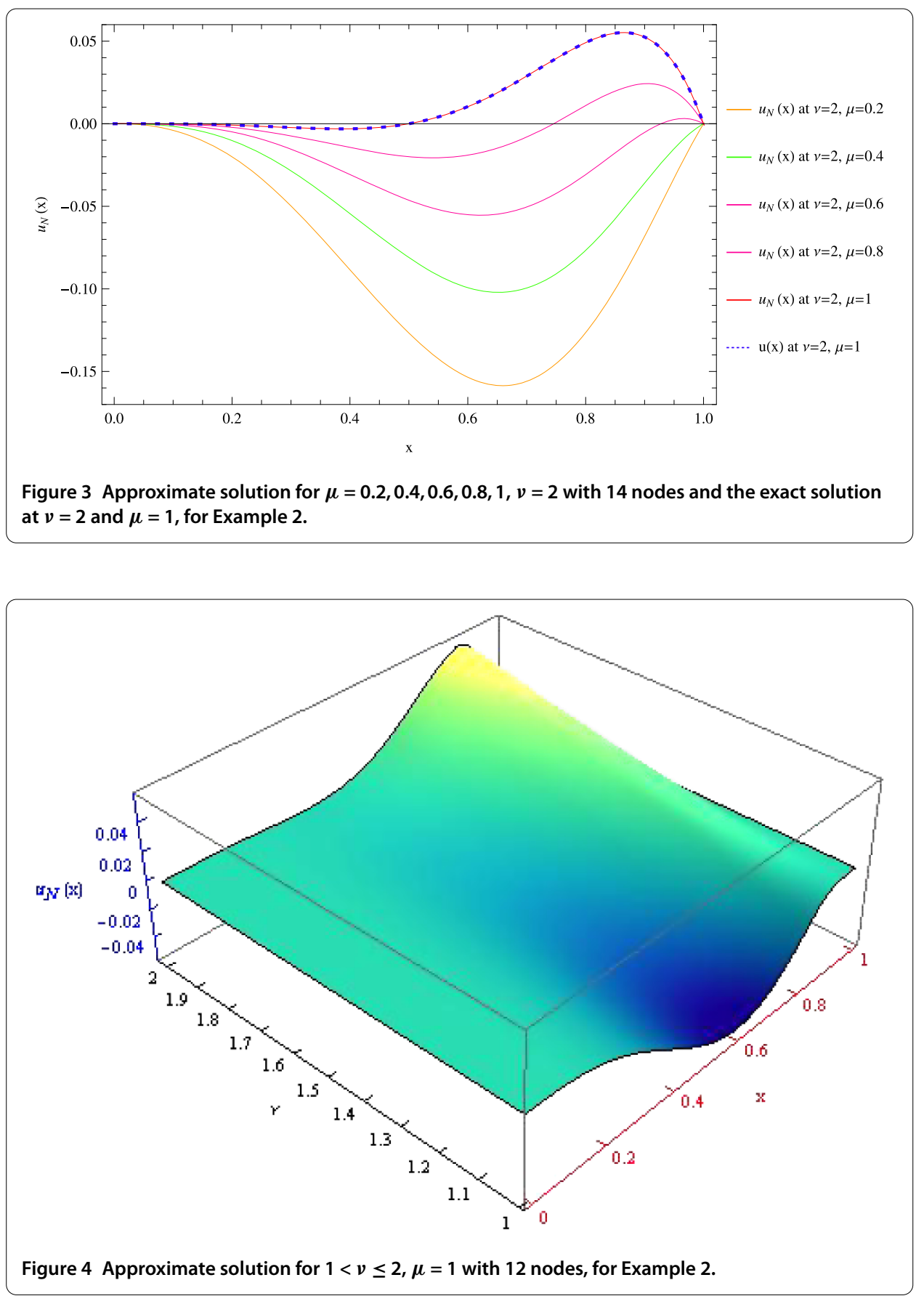

\section{Conclusion}

An efficient and accurate numerical scheme based on the Jacobi-Gauss-Lobatto collocation spectral method is proposed for solving the nonlinear fractional Langevin equation. The problem is reduced to the solution of nonlinear algebraic equations. Numerical examples were given to demonstrate the validity and applicability of the method. The results show that the SJ-GL-C method is simple and accurate. In fact, by selecting a few collocation points, excellent numerical results are obtained. 


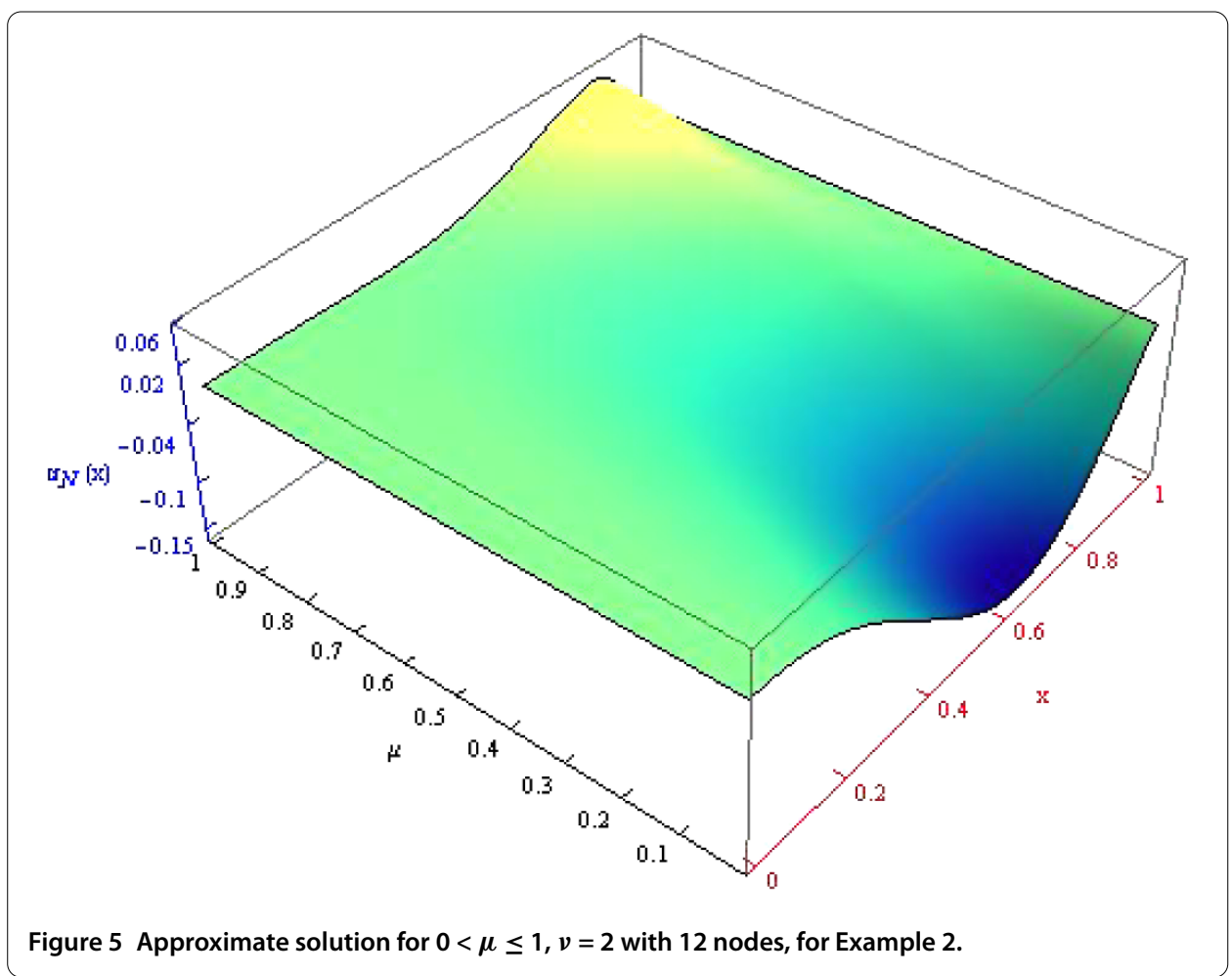

\section{Competing interests}

The authors declare that they have no competing interests.

\section{Authors' contributions}

The authors have equal contributions to each part of this article. All the authors read and approved the final manuscript.

\section{Acknowledgements}

This study was supported by the Deanship of Scientific Research of King Abdulaziz University. The authors would like to thank the editor and the reviewers for their constructive comments and suggestions to improve the quality of the article.

Received: 2 April 2012 Accepted: 30 May 2012 Published: 22 June 2012

\section{References}

1. Magin, RL: Fractional Calculus in Bioengineering. Begell House Publishers, New York (2006)

2. Das, S: Functional Fractional Calculus for System Identification and Controls. Springer, New York (2008)

3. Jafari, H, Yousefi, SA, Firoozjaee, MA, Momanic, S, Khalique, CM: Application of Legendre wavelets for solving fractional differential equations. Comput. Math. Appl. 62, 1038-1045 (2011)

4. Bhrawy, AH, Alofi, AS: The operational matrix of fractional integration for shifted Chebyshev polynomials. Appl. Math. Lett. (2012). doi:10.1016/j.aml.2012.01.027

5. Lotfi, A, Dehghan, M, Yousefi, SA: A numerical technique for solving fractional optimal control problems. Comput. Math. Appl. 62, 1055-1067 (2011)

6. Lakestani, M, Dehghan, M, Irandoust-pakchin, S: The construction of operational matrix of fractional derivatives using B-spline functions. Commun. Nonlinear Sci. Numer. Simul. 17, 1149-1162 (2012)

7. Pedas, A, Tamme, E: Piecewise polynomial collocation for linear boundary value problems of fractional differential equations. J. Comput. Appl. Math. (2012). doi:10.1016/j.cam.2012.03.002

8. Bhrawy, AH, Alofi, AS, Ezz-Eldien, SS: A quadrature tau method for variable coefficients fractional differential equations. Appl. Math. Lett. 24, 2146-2152 (2011)

9. Bhrawy, AH, Alshomrani, M: A shifted Legendre spectral method for fractional-order multi-point boundary value problems. Adv. Differ. Equ. 2012, 8 (2012)

10. Doha, EH, Bhrawy, AH, Ezz-Eldien, SS: Efficient Chebyshev spectral methods for solving multi-term fractional orders differential equations. Appl. Math. Model. 35, 5662-5672 (2011)

11. Doha, EH, Bhrawy, AH, Ezz-Eldien, SS: A Chebyshev spectral method based on operational matrix for initial and boundary value problems of fractional order. Comput. Math. Appl. 62, 2364-2373 (2011)

12. Ahmad, B, Nieto, JJ, Alsaedi, A, El-Shahed, M: A study of nonlinear Langevin equation involving two fractional orders in different intervals. Nonlinear Anal., Real World Appl. 13, 599-606 (2012)

13. Ahmad, B, Nieto, J: Solvability of nonlinear Langevin equation involving two fractional orders with Dirichlet boundary conditions. Int. J. Differ. Equ. 2010, Article ID 649486 (2010). doi:10.1155/2010/649486

14. Chen, A, Chen, Y: Existence of solutions to nonlinear Langevin equation involving two fractional orders with boundary value conditions. Bound. Value Probl. 2011, Article ID 516481 (2011). doi:10.1155/2011/516481 
15. Fa, KS: Fractional Langevin equation and Riemann-Liouville fractional derivative. Eur. Phys. J. E 24, 139-143 (2007)

16. Picozzi, S, West, B: Fractional Langevin model of memory in financial markets. Phys. Rev. E 66, 46-118 (2002)

17. Lim, SC, Li, M, Teo, LP: Langevin equation with two fractional orders. Phys. Lett. A 372, 6309-6320 (2008)

18. Eab, CH, Lim, SC: Fractional generalized Langevin equation approach to single-file diffusion. Physica A 389 2510-2521 (2010)

19. Canuto, C, Hussaini, MY, Quarteroni, A, Zang, TA: Spectral Methods in Fluid Dynamics. Springer, New York (1988)

20. Bhrawy, AH, Alofi, AS: A Jacobi-Gauss collocation method for solving nonlinear Lane-Emden type equations. Commun. Nonlinear Sci. Numer. Simul. 17, 62-70 (2012)

21. Guo, B-Y, Yan, J-P: Legendre-Gauss collocation method for initial value problems of second order ordinary differential equations. Appl. Numer. Math. 59, 1386-1408 (2009)

22. Saadatmandi, A, Dehghan, M: The use of sinc-collocation method for solving multi-point boundary value problems. Commun. Nonlinear Sci. Numer. Simul. 17, 593-601 (2012)

23. Doha, EH, Bhrawy, AH, Hafez, RM: On shifted Jacobi spectral method for high-order multi-point boundary value problems. Commun. Nonlinear Sci. Numer. Simul. 17, 3802-3810 (2012)

24. Doha, EH, Bhrawy, AH: Efficient spectral-Galerkin algorithms for direct solution of fourth-order differential equations using Jacobi polynomials. Appl. Numer. Math. 58, 1224-1244 (2008)

25. Doha, EH, Bhrawy, AH: A Jacobi spectral Galerkin method for the integrated forms of fourth-order elliptic differential equations. Numer. Methods Partial Differ. Equ. 25, 712-739 (2009)

26. El-Kady, M: Jacobi discrete approximation for solving optimal control problems. J. Korean Math. Soc. 49, 99-112 (2012)

27. Doha, EH, Abd-Elhameed, WM, Youssri, YH: Efficient spectral-Petrov-Galerkin methods for the integrated forms of third- and fifth-order elliptic differential equations using general parameters generalized Jacobi polynomials. Appl. Math. Comput. 218, 7727-7740 (2012)

28. Xie, Z, Wang, L-L, Zhao, X: On exponential convergence of Gegenbauer interpolation and spectral differentiation. Math. Comput. (2012, in press)

29. Liu, F, Ye, X, Wang, X: Efficient Chebyshev spectral method for solving linear elliptic PDEs using quasi-inverse technique. Numer. Math. Theor. Meth. Appl. 4, 197-215 (2011)

30. Zhu, L, Fan, Q: Solving fractional nonlinear Fredholm integro-differential equations by the second kind Chebyshev wavelet. Commun. Nonlinear Sci. Numer. Simul. 17, 2333-2341 (2012)

31. Doha, EH, Bhrawy, $\mathrm{AH}$ : An efficient direct solver for multidimensional elliptic Robin boundary value problems using a Legendre spectral-Galerkin method. Comput. Math. Appl. (2012). doi:10.1016/j.camwa.2011.12.050

32. Podlubny, I: Fractional Differential Equations. Academic Press, San Diego (1999)

33. Szegö, G: Orthogonal Polynomials. Am. Math. Soc. Colloq. Pub., vol. 23 (1985)

34. Doha, EH: On the coefficients of differentiated expansions and derivatives of Jacobi polynomials. J. Phys. A, Math. Gen. 35, 3467-3478 (2002)

35. Doha, EH: On the construction of recurrence relations for the expansion and connection coefficients in series of Jacobi polynomials. J. Phys. A, Math. Gen. 37, 657-675 (2004)

36. Doha, EH, Bhrawy, AH, Ezz-Eldien, SS: A new Jacobi operational matrix: an application for solving fractional differential equations. Appl. Math. Model. (2012). doi:10.1016/j.apm.2011.12.031

doi:10.1186/1687-2770-2012-62

Cite this article as: Bhrawy and Alghamdi: A shifted Jacobi-Gauss-Lobatto collocation method for solving nonlinear fractional Langevin equation involving two fractional orders in different intervals. Boundary Value Problems 2012

2012:62.

\section{Submit your manuscript to a SpringerOpen ${ }^{\circ}$ journal and benefit from:}

- Convenient online submission

- Rigorous peer review

- Immediate publication on acceptance

- Open access: articles freely available online

- High visibility within the field

- Retaining the copyright to your article 